\title{
Concepciones de estudiantes y maestros de educación básica en torno a lo sonoro y el espacio geográfico: implicaciones didácticas*
}

\author{
Giovanni Zapata-Cardona** \\ Juan Diego Cardona Restrepo ${ }^{* * * *}$
}

Recibido: 16-07-2020

Aceptado: $21-07-2020$

Citar como: Zapata-Cardona, G. y Cardona Restrepo, J. D. (2021). Concepciones de estudiantes y maestros de educación básica en torno a lo sonoro y el espacio geográfico: implicaciones didácticas. Revista Interamericana de Investigación, Educación y Pedagogía, 14(2), 141-167. https://doi.org/10.15332/25005421.6026

\section{Resumen}

El presente artículo determina las relaciones existentes entre lo sonoro y el espacio geográfico, desde la voz de estudiantes y maestros de educación básica. Dada la naturaleza cualitativa de la indagación, se opta por un estudio de caso de corte instrumental en el que la entrevista y el cuestionario son las técnicas para la recolección de datos. Al analizar los resultados, se encuentra que es posible evidenciar un conjunto de relaciones desde las dimensiones de lo social, lo subjetivo, lo físico y lo holístico, a partir de las cuales se puede pensar lo sonoro como elemento didáctico alternativo y el espacio geográfico como objeto integrador de las disciplinas en la enseñanza de las ciencias sociales en la escuela.

\footnotetext{
* Artículo derivado de la tesis titulada Lo sonoro del espacio urbano. Aportes a la enseñanza de las ciencias sociales en la escuela para la Maestría en Ciencias: Innovación en Educación de la ITM Institución Universitaria (2020).

** Licenciado en Educación Básica con Énfasis en Ciencias Sociales, magíster en Ciencias e Innovación en Educación del Instituto Tecnológico Metropolitano (ITM) y docente adscrito a la Secretaría de Educación de Medellín.

Correo electrónico: giozapacar@gmail.com

ORCID: https://orcid.org/0000-0003-4694-5803

Google Académico: https://scholar.google.com/citations?user=5OuAGc8AAAAJ\&hl=es

*** Licenciado en Educación básica con énfasis en Ciencias Naturales. Investigación en la enseñanza y el aprendizaje de las ciencias experimentales. Doctor en Educación. Integrante del Grupo de Investigación Innovaciencia, Universidad de Antioquia, Colombia.

Correo electrónico: jcardonarestrepo@gmail.com

ORCID: https://orcid.org/0000-0002-7194-678X

Google Académico: https://scholar.google.es/citations?hl=es\&user=X0T3rOAAAAAJ
} 
Palabras clave: educación, enseñanza de las ciencias sociales, geografía, escucha, sonido, ciencias sociales.

\title{
Conceptions of Students and Teachers of Basic Education Around Sound and Geographic Space: Didactic Implications
}

\begin{abstract}
This article aims to determine the existing relationships between sound and geographic space, from the voice of students and teachers of basic education. Given the qualitative nature of the research, an instrumental case study was chosen in which the interview and the questionnaire are the techniques for data collection. The data analysis showed that it is possible to evidence a set of relationships from the dimensions of the social, the subjective, the physical, and the holistic, from which it is possible to think about sound as an alternative didactic element and about geographic space as an integrating object of disciplines in the teaching of social sciences at school.
\end{abstract}

Keywords: education, social sciences teaching, geography, listening, sound, social sciences.

\section{Concepções de alunos e professores da educação básica sobre o espaço sonoro e geográfico: implicações didáticas}

\section{Resumo}

Este artigo tem como objetivo verificar as relações existentes entre o espaço sonoro e geográfico a partir da voz de alunos e professores da educação básica. Dada a natureza qualitativa da investigação, opta-se por um estudo de caso instrumental em que a entrevista 
e o questionário constituem as técnicas de coleta de dados. Ao analisar os dados, verifica-se que, é possível evidenciar um conjunto de relações com base nas dimensões do social, do subjetivo, do físico e do holístico, a partir das quais se pensa o som como elemento didático alternativo e o espaço geográfico como objeto integradordas disciplinas do ensino de ciências sociais na escola.

Palavras-chave: educação, ensino de ciências sociais, geografia, escuta, som, ciências sociais.

\section{Introducción}

La enseñanza de las ciencias sociales en la escuela, desde una perspectiva crítica, parte de considerar el desarrollo de cuatro finalidades: la construcción del conocimiento social, la formación del pensamiento social, la comprensión de la realidad social y la formación ciudadana y democrática (Benejam, 2004; Pagés, 2007; Santisteban, 2011). Estas finalidades son elementos clave para la adopción de un nivel alto de criticidad, a partir del cual los estudiantes asumen la comprensión de las realidades a través del análisis y problematización de lo social como algo dinámico y cambiante, resignificando y transformando sus conocimientos en pro de la calidad de vida de las comunidades a las que pertenecen. Así lo establecen Mejía y Mejía (2015), al señalar que el propósito de la escuela crítica "es que el estudiante sea cada vez más consciente de su propio sistema de valores, sea capaz de hacer una reflexión crítica de lo que piensa, quiere y pueda pensar posibles alternativas ante los sucesos que aborda" (p. 418).

Desde este punto de vista, para el caso colombiano, a nivel curricular se encuentran propuestas que invitan a la innovación de los procesos pedagógicos en diversos documentos oficiales, tales como los Lineamientos Curriculares, los Estándares Básicos por Competencias y, recientemente, el documento de los Derechos Básicos del Aprendizaje. En estos, se entiende la acción de innovar 
como "trabajar en un horizonte de mejora continua para cuantos intervienen en el acto formativo y demostrar que los implicados en tal acción logran los objetivos y dominan las competencias básicas que se estiman más valiosas" (Domínguez, Medina y Sánchez, 2011, p. 66). Además de la integración de una mirada interdisciplinaria de lo social desde múltiples focos y dimensiones; condición sine qua non para la selección y determinación de los contenidos - desde lo conceptual, procedimental y actitudinal- que se deben enseñar en la escuela.

De esta manera, se presta atención a las relaciones causales de la enseñanza y el aprendizaje, un acto didáctico, a veces, carente de herramientas diversas y modos alternativos para promover la adquisición de los saberes y habilidades. Asimismo, lleva a que los educandos se instalen en una posición desde la cual, la realidad social es vista bajo una mirada simplista que excluye la noción de la misma como construcción social y establece barreras en la formación de un pensamiento social holístico, entendido este como "una habilidad que construyen los sujetos y que les permite analizar la sociedad en la que viven, generando interrogantes, identificando relaciones espacio-temporales, problematizando las acciones y transformando sus visiones y significados" (Garzón, 2017, p. 50), que no puede adquirirse por mera transmisión, sino que exige un aprendizaje activo, en el que los estudiantes dominen las habilidades de indagación a través de las fuentes de información.

En consecuencia, se retoman los trabajos de la profesora Pulgarín (2002), quien, a partir del estudio de las acepciones que adquieren el espacio geográfico, el lugar, el paisaje, la región, el medio geográfico, el geosistema y el territorio, defiende la postura de que dichos conceptos se erigen como posibilidades al momento de repensar la integración de las ciencias sociales en el ámbito escolar, incluso la transversalidad del currículo, puesto que se da un cambio de paradigma del espacio geográfico; de un espacio físico inmutable a un espacio social producido y transformado. Según Espinal y Pulgarín (2008), lo anterior ha posibilitado que la geografía se ubique tanto en el campo de las ciencias naturales como en el de las ciencias sociales, lo que permite considerar 
la diversidad de disciplinas que se leen en su contexto y la importancia de su enseñanza desde una visión interdisciplinaria.

De este modo, valdría la pena la pregunta en torno a las formas y los medios en que se pueden llevar al plano concreto estrategias que aboguen por la integración de las ciencias sociales y sus vínculos con otras disciplinas a partir de las posibilidades ofrecidas por el espacio geográfico, como constructo hecho desde la complejidad. Ante esto, aparece un horizonte conceptual amplio en el trabajo, con líneas de investigación que permitan dilucidar cómo el ser humano establece relaciones con el entorno a través del sonido, pero, a la vez, la incidencia de lo sonoro en la vida social, provocando con ello que emerjan y tomen fuerza diversos conceptos y categorías de estudio, como paisaje sonoro (Schafer, 1969), auralidad (Sui, 2000), acustemología (Feld, 1996), por citar solo algunos.

Lo anterior toma fuerza, ya que constituye una crítica y reconfiguración que se ha querido adelantar frente a la supremacía dada a la vista respecto a otros sentidos. Así, por ejemplo, Cosgrove (2002) denuncia cómo el pensamiento occidental ha llegado a negar la importancia de los otros sentidos humanos al momento de leer y comprender el espacio en su acepción de paisaje. Del mismo modo, Zigor (2017) aduce que "la invención del sistema de representación de la perspectiva situó al ojo en el centro del mundo de los sentidos" (p. 23), lo cual ha ocasionado que los estudios sobre lo sonoro tengan vieja data en las disciplinas sociales. Esta situación ha venido cambiando, pues paulatinamente lo sonoro ha sido un objeto emergente dentro de la configuración de disciplinas como la geografía. Así lo admite Fortuna (2009), al mencionar la contribución de Paul Rodaway, quien a partir del concepto de geografías auditivas sitúa un "campo específico de análisis de la experiencia sensible y de las propiedades acústicas del medio ambiente" (p. 44). De hecho, al indagar sobre este asunto, es constante que aparezcan registros que remiten, principalmente, al concepto de paisaje sonoro, cuestión que debería tomarse desde una lectura bajo la perspectiva geográfica, tratando de dilucidar el papel de otras acepciones del espacio geográfico en dicha relación. 
De esta manera, las relaciones entre lo sonoro y el espacio geográfico se erigen como elementos susceptibles de ser analizados desde diversas concepciones entendidas como:

[...] aquellas ideas que se constituyen en teorías implícitas que los seres humanos forman en sus mentes como medio de aproximación a diversas situaciones de la vida cotidiana, así como un modo de organizar todos aquellos conceptos del mundo del que se hace parte y darle así sentido, las cuales definen en cierta medida la manera de afrontar estas situaciones y actuar en el mundo. (Sánchez, 2015, p. 269)

En el marco de una reflexión que genere aportes a la didáctica de las ciencias sociales, a partir de la voz de estudiantes y maestros, se amplía el panorama respecto al trabajo desde lo sonoro. Así, la pregunta que sirve de derrotero es: ¿cuáles son las concepciones de estudiantes y maestros de educación media en torno a la relación entre lo sonoro y el espacio geográfico? Este es el principal objetivo de la investigación que se presenta a continuación.

\section{Metodología}

\section{Diseño}

Al instalarse la propuesta en la perspectiva cualitativa, acoge el enfoque hermenéutico como aspecto que pone de manifiesto la importancia de buscar el sentido en las concepciones declaradas de los participantes. Este proceso está sustentado en el estudio de caso como hoja de ruta, el cual es definido por Stake (2005) como el estudio de la particularidad y la complejidad de un caso singular. De esta manera, se retoman los distintos momentos del método a partir de lo establecido por Martínez (1998): fase preactiva, fase interactiva y fase posactiva. 


\section{Participantes}

Los informantes son estudiantes y maestros de una institución educativa privada de la ciudad de Medellín, Colombia. Sobre los estudiantes, se indica que hacen parte de un conjunto de individuos con edades entre los 15 y 17 años, residentes en el nororiente de la ciudad. La muestra del tipo no probabilístico está conformada por seis estudiantes del grupo 10.1, quienes por su diversidad en el nivel de desempeño (bajo, básico y alto) respecto a los conceptos, procedimientos y actitudes específicos del área de ciencias sociales, estuvieron en condiciones de brindar información variada para describir las relaciones entre lo sonoro y el espacio geográfico. Estas condiciones, junto al hecho de que el investigador es quien orienta el área, al conocimiento que tiene sobre la población, a la intensidad horaria del área, a las condiciones materiales ofrecidas por la institución y al manejo ético desde el consentimiento informado, posibilitaron que se presentara un marco favorable para llevar a cabo, de manera oportuna, el proceso investigativo frente al cumplimiento del objetivo planteado.

Respecto a los cinco maestros de ciencias sociales participantes, se debe decir que constituyen dos grupos heterogéneos: el primero, conformado por dos maestros que llevan entre diez y cinco años en el colegio, con edades entre los 30 y 37 años, quienes han cursado las carreras de grado en historia y en licenciatura en geografía e historia; el segundo, conformado por tres maestros, entre los 26 y 28 años, egresados de la licenciatura en educación básica con énfasis en ciencias sociales de la Universidad de Antioquia.

\section{Técnicas}

Se utilizaron dos técnicas: el cuestionario para los estudiantes y la entrevista semiestructurada para los maestros. El cuestionario estaba compuesto por nueve preguntas abiertas, de esta manera los estudiantes podían responder de acuerdo con sus concepciones y en sus propias palabras. Por su parte, la entrevista semiestructurada fue la base para que los maestros pudieran expresar sus percepciones frente a los temas de investigación. 


\section{Proceso de análisis}

Una vez recolectada la información, esta fue sistematizada en una tabla conformada por dos columnas: la primera, donde se realizó la categorización y la recategorización, y se registraron las anotaciones especiales; la segunda, en la que se transcribieron literalmente las respuestas de los estudiantes y maestros. Con la transcripción de los datos y acogiendo los principios del análisis de contenido, se determinaron las unidades de registro, que, en palabras de Bardin (1996), "son las unidades de significado que se han de codificar y que se corresponden con el segmento de contenido que será necesario considerar como unidad de base con miras a la categorización" (p. 79). Específicamente, se definieron dos unidades de registro o de análisis: la palabra y el tema, ambas usadas para identificar los segmentos de las ideas de los participantes susceptibles de ser categorizados de acuerdo con la tipología de categorías establecida en la tabla 1.

Tabla 1. Marco de referencia para el análisis de las concepciones.

\begin{tabular}{|c|c|c|c|}
\hline Ámbito & Categoria & Subcategoría & Código \\
\hline \multirow{10}{*}{$\begin{array}{l}\text { I. Relaciones entre } \\
\text { lo sonoro y el } \\
\text { espacio geográfico }\end{array}$} & \multirow{3}{*}{$\begin{array}{l}\text { I. A. Dimensión } \\
\text { física }\end{array}$} & Contaminación acústica & I.A.1 \\
\hline & & Ruido & I.A.2 \\
\hline & & $\begin{array}{l}\text { Elementos naturales (aplica solo para los } \\
\text { estudiantes) }\end{array}$ & I.A.3 \\
\hline & \multirow{3}{*}{$\begin{array}{l}\text { I. B. Dimensión } \\
\text { social }\end{array}$} & Patrimonio sonoro & I.B.1 \\
\hline & & Identidad sonora & I.B.2 \\
\hline & & Territorio sonoro & I.B.3 \\
\hline & \multirow{3}{*}{$\begin{array}{l}\text { I. C. Dimensión } \\
\text { subjetiva }\end{array}$} & Sonidos agradables & I.C.1 \\
\hline & & Ruido & I.C.2 \\
\hline & & Paisaje sonoro & I.C.3 \\
\hline & $\begin{array}{l}\text { I. D. Dimensión } \\
\text { holística }\end{array}$ & $\begin{array}{l}\text { Interacción de las acciones humanas con lo } \\
\text { sonoro }\end{array}$ & I.D.1 \\
\hline \multirow{3}{*}{$\begin{array}{l}\text { II. Proceso } \\
\text { didáctico (aplica } \\
\text { solo para los } \\
\text { maestros) }\end{array}$} & \multirow{3}{*}{ II. A. Enseñanza } & Contenido & II.A.1 \\
\hline & & Estrategia & II.A.2 \\
\hline & & Finalidad & II.A.3 \\
\hline
\end{tabular}




\section{Resultados y discusión}

Es importante mencionar que, para la elaboración del cuestionario y la entrevista, hubo un proceso previo de revisión documental, que posibilitó determinar las principales categorías que tuvieron en cuenta la relación aquí expuesta desde lo teórico. Esto sirvió para el análisis referido en este apartado. Los resultados se presentarán a partir de la división por grupo de participantes.

\section{Desde el punto de vista de los estudiantes}

A partir de la información aportada por los seis estudiantes de grado décimo que respondieron el cuestionario, se han identificado 56 unidades de análisis (de aquí en adelante UA), de las cuales, frente a la relación entre lo sonoro y el espacio geográfico, el $40 \%$ se refiere a la dimensión física, un $30 \%$ a la dimensión social y otro $30 \%$ a la dimensión subjetiva; aclarando que no se han logrado obtener enunciados que hicieran referencia a la categoría denominada Dimensión holística (código I. D.). Sobresale, por encima de las demás, la categoría I. A. Dimensión física $(n=22)$, situación que podría esperarse a priori, ya que durante la revisión documental se encontró bastante bibliografía que contenía la relación entre el espacio geográfico y lo sonoro desde la perspectiva de mapas de ruido como herramienta de medición del sonido en su acepción de contaminante ambiental.

Se debe señalar que a la pregunta que solicitaba una descripción del espacio geográfico de la ciudad, la mayoría de estudiantes se remitió a conceptos desde el ámbito físico de la geografía, resaltando aquellos elementos naturales que conforman el relieve de la ciudad:

En mi ciudad hay muchas montañas, es una ciudad que forma un valle estrecho, así que se puede ver todo el paisaje de la ciudad independientemente de donde estés ubicado. Hay amplias y variedad de construcciones 
en el centro, edificios de gran altura, parques de todo tipo; a pesar de que hay mucha construcción en las laderas, aún quedan muchas zonas verdes. Las condiciones climáticas son cálidas y hay una buena organización de los espacios. (UA 13)

Lo anterior, evidencia el nivel de apropiación que poseen los estudiantes, a quienes, en un grado avanzado de la educación media, se les dificulta declarar un espacio geográfico producto de las relaciones dinámicas entre sociedad y naturaleza, concibiéndolo simplemente como un conjunto de elementos naturales establecidos en un espacio delimitado.

Ahora bien, si nos trasladamos al ámbito de lo que sugieren las relaciones aquí problematizadas, se encuentra que el punto común de la relación entre lo sonoro y el espacio geográfico desde la dimensión física, se establece a partir de las subcategorías de ruido y contaminación acústica. De acuerdo con las concepciones de los estudiantes, en cierta medida se confirma esta correlación de elementos:

Al parecer, a todos en esta ciudad les encantan gritar, siempre que salgo a la calle encuentro gente gritando, el sonido de las bocinas con las que venden ciertos productos y las de los carros. A la ciudad la caracteriza el alto flujo de ruido. (UA 8)

Para entender las múltiples aristas de la relación aquí expuesta, hay que mencionar que las concepciones de los estudiantes tienden a ser más complejas, en la medida en que se van integrando en las mismas otros aspectos como lo subjetivo. De igual forma, como se verá más adelante, se complejizan las concepciones cuando se establecen vínculos entre lo sonoro y lo social. Esto evidencia que dichas concepciones tienen un origen implícito o intuitivo, ya que se corresponden con procesos básicos de aprendizaje de los cuales, casi siempre, los alumnos no son conscientes de que surgen de sus experiencias personales, puesto que muchos, al momento de responder al cuestionario, 
manifestaron que, pese a que es algo que no está alejado de su cotidianidad, es una reflexión nueva para ellos.

A pesar de que el estudiantado utiliza en sus enunciados nociones muy genéricas respecto al componente físico del sonido, logran identificar el transporte como principal fuente generadora de ruido, coincidiendo con Noriega (2017):

La contaminación acústica puede propagarse de dos formas principales, por vía aérea y por medio de vibraciones. Por ejemplo, el ruido de transportes en una ciudad puede afectar a las zonas residenciales circundantes a grandes vías de comunicación o viviendas cerca de aeropuertos, por la vía de transmisión aérea, es decir, el sonido se transmite de manera directa desde la fuente hasta los humanos a través del aire, y la fuente del ruido es identificada por las personas directamente. (Noriega, 2017, p.76)

De esta manera, los estudiantes no alcanzan a definir una distinción plena entre sonido y ruido, entendido este último como algo indeseable dentro de la dinámica perceptiva respecto a la ciudad. Se resaltan los tipos de sensaciones que produce dicha exposición, además ubicando espacialmente focos de contaminación en la ciudad: "muchas veces depende del sector, en el centro por ejemplo es el de los pitos de los medios de transporte, ya que crean congestión, y en el barrio me molesta los vecinos que son muy escandalosos" (UA 23).

Cabe destacar, que, a pesar de configurar el ruido, también dentro del discurso del estudiante aparecen enunciados que apelan a elementos naturales, los cuales, en cierta manera, enmascaran el nivel de ruido al que se está expuesto: "Hay algo muy particular y es que en la Avenida Oriental por las tardes se escuchan unos loros que están en los árboles, eso rompe un poquito con la congestión de ruido que caracteriza al centro" (UA 19).

La mayoría concibe lo sonoro y el espacio desde una dupla de ruido versus sonido agradable, refiriendo este último a aspectos 
donde prima la naturaleza por encima del factor antrópico. Es decir, el $90 \%$ de los estudiantes no ubicaría los sonidos agradables en el ámbito urbano, sino en el rural. De acuerdo con Quintero y Recuero (2018), el paisaje sonoro de un lugar se liga al tipo de actividad que allí se establece.

En las concepciones se alcanza a intuir una referencia a elementos articuladores de la perspectiva física del sonido, como el nivel de presión sonora, el espectro, el carácter temporal y la localización, develando una relación siempre con el ruido. Aquí vale la pena acudir a lo planteado por Boned (2011): "Cuando el fondo auditivo invade por completo el espacio sonoro (cuando el ruido ambiental es demasiado fuerte), la selección, la inteligencia del espacio, ya no es posible, y la escucha resulta perjudicada." (p. 5). La mayoría de los estudiantes reconoce en su discurso algunas fuentes sonoras dentro de la amalgama de la ciudad.

La figura del Gobierno aparece como aquel agente importante en la gestión del ruido, un problema en el que las soluciones, según denuncia Cuervo (2015), parecen insuficientes, puesto que, al seguir realizándose con base en las mediciones y el levantamiento de mapas de ruido, "no contemplan el contexto ni las relaciones con los habitantes y, además, las normativas solo suelen incluir la presión sonora como un indicador aislado de la percepción" (p. 94). Esta situación aparece implícitamente en lo expresado por los estudiantes: "En cuanto a lo ambiental, me parece terrible, no hay consciencia, las personas siguen creyendo que es un invento la contaminación y el propio Gobierno no es consciente de que la situación no cambia" (UA 10).

Por otro lado, la categoría I. B. Dimensión social y la categoría I. C. Dimensión subjetiva ocupan posiciones destacadas, con una frecuencia igual $(n=17)$. Dichas categorías, en un alto porcentaje, se cruzan entre sí en el discurso de los estudiantes, quienes por un lado destacan que lo sonoro se convierte en un elemento que brinda información socioespacial, pero a la vez aducen se encuentra íntimamente ligado a la percepción que cada sujeto realice, ejemplo de ello es la categoría de ruido. 
Frente a la dimensión social, es posible afirmar que entran en juego cuestiones remitidas a la identidad, el patrimonio y la caracterización de las sociedades a partir de lo sonoro. Según Mejía (2012), los sonidos propios de una comunidad, producto de la relación sujetoentorno y sujeto-sujeto, deben ser reconocidos y expuestos por sus actores principales para que, de esta manera, las condiciones que acompañan la configuración de la identidad se vuelvan visibles ante individuos externos. En la siguiente expresión, un estudiante alude a las sonoridades propias de un lugar determinado en cierta época del año, donde la música se convierte en eje generador de sentido del lugar; como diría Duffy (2000), un sentimiento de pertenencia y de vinculación identitaria a nivel comunitario. Sonidos que se convierten en memoria colectiva de las comunidades $y$, en esa medida, que pueden reconocerse como patrimonios inmateriales: "Están los sonidos de épocas como diciembre, donde en la mayoría de barrios de la ciudad todo el mes se escucha música parrandera y también en Feria de Flores en el mes de agosto" (UA 5).

En cuanto a la pregunta sobre si consideran que los sonidos podrían brindar información de la dinámica socioespacial, partiendo de que todo sonido "está concretamente determinado por las actividades del hombre sobre la naturaleza, en lo referente a sus labores diarias, domésticas o especializadas, en el campo o en las urbes; en el trabajo en las fábricas o en las pequeñas empresas, etc." (Cárdenas y Martínez, 2015, p. 133), uno de los estudiantes responde:

El desorden que maneja la ciudad es abismal y si te pones a detallar los ruidos de la ciudad son eso, caos. Los carros pitan en un trancón como si eso fuera a solucionar algo, solo se impacientan y comienzan a ser muy ruidosos, las personas con un mínimo desacuerdo comienzan a gritarle a otros, se sobrepone la voz de todos y es desesperante, es un caos y a veces eso sí es la ciudad. Eso habla de nuestro sistema cultural. (UA 11)

Unidades de análisis como esta, que pueden ser ubicadas en varias subcategorías, ponen de manifiesto, por un lado, el ruido 
ambiental $y$, por otro, las sensaciones que le producen al sujeto estar expuesto a dicho ruido; asimismo, la referencia a un sistema cultural de valores que tiene incidencia directa en la forma en que producimos la espacialidad. Siguiendo a Westerkamp (1988, citado en Cárdenas y Martínez, 2015), todas y cada una de estas labores son productoras de sonido o fuentes sonoras que le comunican al ser humano, de una manera particular y a través del sonido o ruido, los acontecimientos que suceden a su alrededor y le indican que él es productor de sonidos; por lo tanto, tiene injerencia en la calidad de su espacio sonoro.

En este sentido, los estudiantes ubican el sonido del parque automotor como la fuente más característica de la actividad urbana:

Los sonidos característicos de la ciudad son el sonido de los buses, de los vendedores ambulantes. No hay forma de ubicar estos sonidos en algún tiempo y época del año, ya que siempre están presentes desde el principio hasta fin de año. (UA 44)

La anterior unidad de análisis alerta, además, sobre la manera en que se ve la actividad urbana como algo constante en el tiempo; pero, ¿será que verdaderamente las sonoridades urbanas permanecen estáticas? Para agregar más elementos a esta reflexión, se pueden identificar otros aspectos que permiten inferir la forma como los estudiantes van incorporando y correlacionando nuevos conceptos que van permitiendo una mejor compresión del asunto desde lo espacio-temporal:

El sonido de la ciudad varía dependiendo de la hora, es decir, en el día se puede evidenciar bastante ruido ya sea por vehículos o artefactos tecnológicos, pero en la noche se puede sentir un ambiente más de tranquilidad, debido a que la gran mayoría de las personas tiene que madrugar al siguiente día. (UA 53)

En cuanto a lo espacial, aparecen distintos elementos: el centro como abrumador sonoro o ruidoso, los barrios con casas donde 
ponen la música a alto volumen y otros barrios, como se evidencia al sur de la ciudad, con más calma. Así, el espacio público va determinando un tipo de relaciones, donde el ruido se convierte en intruso. "La variabilidad sobre la percepción del ruido se explica por el hecho de que el sonido es un intruso solo cuando desajusta nuestros hábitos o nos impide cumplir con nuestra disposición personal de actividades" (Domínguez, 2015, p. 120). Lo anterior, se ve reflejado en declaraciones como esta: "Me estresa eso, sí el sonido, más que todo de las zonas urbanas, como el de los pitos de los carros, la música exageradamente fuerte. Prefiero estar con mis audífonos puestos cuando estoy en espacios públicos" (UA 42).

Se aprecia la configuración de una producción y percepción sonora que demanda acciones por parte de los sujetos involucrados, sea a través de violencia, resignación, diálogo, denuncia, o mediante la producción de un sonido elegido que compita y enmascare el recibido involuntariamente: "Lo peor para mí es cuando estoy en un lugar público que hay gran cantidad de fuentes de ruido como cantantes, vendedores ambulantes tratando de gritar más duro, como si con eso vendieran más que otros" (UA 55).

Los argumentos, unos más simples y otros con tendencia a ser más complejos, expresan la necesidad de abordar la comprensión del espacio sonoro urbano desde una perspectiva de la convivencia; el sonido inconcebible sin la presencia del otro y el silencio absoluto como un imposible.

Ahora bien, respecto a la dimensión subjetiva, se establece una experiencia estética en la que, fundamentalmente, prevalecen aspectos de desagrado y molestia frente al sonido que envuelve la vida en la ciudad. En menor medida, se encuentran cuestiones positivas, como se evidencia en lo expresado por un estudiante, "La lluvia, el viento y en sí los sonidos que produce la naturaleza me calman" (UA 7).

Sin embargo, estas concepciones de valoración tienden a visiones más complejas desde la dimensión subjetiva, debido a que se aprecia que se está empezando a considerar cómo lo sonoro 
influye en el modo de apropiar el espacio: "Siento que los sonidos influyen mucho en la forma que nos sentimos respecto a un lugar. Si los sonidos crean un lindo ambiente, es probable que nos sintamos en paz. Por el contrario, la congestión auditiva puede intranquilizarnos bastante" (UA 33).

A partir de las concepciones de los estudiantes se puede inferir que estas son limitadas y poco consistentes, respondiendo a ciertas generalidades que, aunque ponen de manifiesto una dinámica social, no trascienden más allá del hecho de describir el ruido, algunos de sus efectos, su ubicación y su fuente. De esta manera, se limitan a develar la articulación global que se ponga en escena.

En síntesis, es posible afirmar que, desde la voz de los estudiantes, sus enunciados remiten a ciertas generalidades. La primera de ellas, que corresponde a la relación desde lo físico, está sustentada en la visión de lo sonoro como ruido causante de contaminación acústica, especialmente en las ciudades, lo que genera no solo una incidencia negativa en la salud de la población, sino también desde lo subjetivo, al pensar la dicotomía espacial: urbano/ruido versus rural/sonidos agradables en relación con lo natural.

En el estudiantado hay poca presencia del concepto de identidad sonora, solo en un registro de análisis se logró encontrar relación entre la identidad y lo sonoro, y, por ende, lo cultural; sin embargo, la mayoría de estudiantes coincidían en indicar que a través de lo sonoro era posible identificar la dinámica socioespacial. Al ahondar en esta situación, desde una perspectiva reduccionista, estos dirigen la atención a los sonidos que genera la actividad humana, mas no se trasciende a otro tipo de vertientes.

Respecto a lo social, se pudo ver que hay un reconocimiento indirecto de las relaciones de poder manifiestas desde el ruido en el espacio, mediante la dupla público/privado. Relaciones de conflicto en situaciones de tráfico, de ventas ambulantes, de convivencia en residencias, barrios con situaciones como el volumen alto de la música o posturas de evitación con artefactos tecnológicos 
en la reclamación de ambientes naturales, definidos como agradables o silenciosos.

Finalmente, hay que apuntar que en el conjunto de concepciones declaradas no apareció ninguna que identifique exclusivamente la categoría I. D. Dimensión holística, que contenga en el discurso una interacción de las acciones humanas con lo sonoro desde la triada de lo social, lo subjetivo y lo físico.

En cuanto a esta situación, surgió la necesidad de girar el foco de atención y ubicarlo en el maestro. Aunque en lo declarado por el estudiantado se lograron identificar aspectos que posibilitaron dar solidez a la propuesta de convertir en objeto didáctico lo sonoro del espacio para movilizar la comprensión de la complejidad de lo urbano, resulta importante considerar la perspectiva del agente responsable de generar las condiciones para la enseñanza.

\section{Desde el punto de vista de los maestros}

A partir de la información aportada por los cinco maestros de ciencias sociales de la institución educativa, en la técnica de la entrevista se identificaron $44 \mathrm{UA}$, de las cuales, frente a la relación entre lo sonoro y el espacio geográfico, el $38 \%$ se relaciona con la dimensión subjetiva, un $15 \%$ con la dimensión social, otro $8 \%$ con la dimensión física y un $9 \%$ con la dimensión holística. Por su parte, la categoría que se refiere a las condiciones de enseñabilidad de lo sonoro del espacio geográfico ocupa un $30 \%$.

De igual forma, se hace referencia a las subcategorías definidas como Sonidos agradables I.C.1, Ruido I.C.2 y Experiencias I.C.3, con el objetivo de establecer aquellos elementos que posibilitaron la relación con el concepto de lugar, a partir de la geografía de la percepción, como información que llega por medio de los sentidos y que es valorada según la historia personal del sujeto; y trazar una línea muy disímil que conecte la dimensión social desde el componente cultural con la acepción de paisaje. 
En primer lugar, se encontró que los docentes destacan el proceso de la percepción en las maneras de leer el espacio:

A partir de lo sonoro puede ser posible percibir aspectos característicos de determinado espacio geográfico por medio de un sentido (la escucha), que ha sido históricamente relegado para realizar ejercicios de indagación/ investigación, dando prioridad a lo que la vista otorga. (UA 58)

Lo anterior, configura el énfasis en un aspecto epistemológico que se incluye en la problematización de las tensiones que originaron la presente pesquisa y es, justamente, el papel que ha tenido, por mucho tiempo, el sentido de la vista dentro de la perspectiva occidental como única forma válida de acceder al conocimiento. Además, el pensamiento occidental ha perpetuado en el tiempo una suerte de jerarquía de los sentidos, coincidiendo con Zigor (2017), quien afirma que "la invención del sistema de representación de la perspectiva situó al ojo en el centro del mundo de los sentidos" (p. 23).

Frente a la distinción entre sonido y ruido, los maestros indicaron planteamientos similares, determinados por la subjetividad: "El sonido sería la categoría general dentro de la cual se incluiría el ruido. El sonido podría ser todo lo que percibimos mediante el sentido de la audición y el ruido sería una interpretación subjetiva de dicha percepción auditiva" (UA 60). Así, desde los criterios de la subjetividad, la capacidad intrusiva de un sonido estará determinada por lo que cada quien percibe como incómodo o indeseable.

Por otra parte, la distinción se basa en la capacidad de evidenciar las fuentes que están produciendo el sonido:

No, yo asocio el ruido como lo que perturba, lo que no tiene armonía, desconcentra, por otro lado, para mí el sonido sería todo lo contrario, aquello que tranquiliza o que al menos se es posible de decodificar e interpretar. (UA 69) 
En torno a la pregunta por la acepción del espacio geográfico, dentro de la cual ubicarían la relación con lo sonoro, los maestros manifestaron: "Con la acepción subjetiva, porque la percepción o creación de sonido va sujeta a las emociones, cultura e incluso a los elementos percibidos del mundo natural" (UA 77).

Particularmente, los profesores reconocen la dimensión del paisaje como elemento articulador, ubicado en el ámbito del espacio sonoro, susceptible de ser leído desde los múltiples sentidos. Cabe anotar, que también se hace referencia a otras acepciones, como la de región geográfica: "Con la acepción de región cultural, en el sentido en que a través de esta se tendría mucho en cuenta las conjunciones presentes dentro de determinadas regiones culturales desde la dimensión antropológica" (UA 83).

De esta manera, se presenta el concepto de región como una unidad del espacio con características que la identifican y diferencian de otras, en una suerte de homogenización, en términos culturales, donde lo sonoro hace presencia desde el registro cultural, referido al patrimonio inmaterial, que se traduce en identidad.

Cabe mencionar que las concepciones de los maestros emergen de un encuentro de conocimientos en torno a lo pedagógico, lo disciplinar y lo didáctico, una triada en interacción que actúa conjugando un saber fundante de su práctica. Sin embargo, para el caso que sugiere la última pregunta analizada, surgen ideas que destacan la importancia que adquiere para el docente la apropiación de los principios epistemológicos, teóricos y metodológicos de su área, desde las indagaciones de carácter científico, que posibilitan configurar su discurso en el marco de la reflexión espacio/ espacialidad. Esta cuestión, indudablemente, se verá reflejada al momento de dar un tratamiento adecuado a los fenómenos, sistemas y procesos sociales al interior del aula.

Con esto, se emprenden los vínculos desde lo social. Los maestros, al igual que los estudiantes, coincidieron en concebir lo sonoro como un elemento que da cuenta de la dinámica socioespacial: 
Podría suponer una fuente de información, en la medida en que da cuenta de las dinámicas de x lugar o espacio concreto mediante su acústica, donde se podría tomar como fuente de insumo para hacer registro y acopio de sonidos que puedan retratar o sirvan para hacer paragón o ponderación respecto a otros insumos y datos empíricos en otro tipo de unidad de registro. (UA 82)

Se pasa, entonces, de un plano subjetivo, en el que las condiciones psicológicas del sujeto se ponen en juego para significar lo que escucha, hacia un plano de lo social, donde el sonido se erige como fuente de información que indica "la relación entre los espacios, las actividades, los materiales y los sonidos asociados a esos espacios y generados como consecuencia de esas actividades" (Alonso-Cambrón, 2005, p. 40).

Asimismo, las concepciones declaradas por los docentes hacen mención al papel que posee lo educativo, pues se torna claro que, si no hay una cultura auditiva que promueva la escucha como fuente de conocimiento, difícilmente el sujeto podrá reconocer elementos de sentido y significación en el espacio sonoro que habita. "Creo que por medio de la educación se puede lograr dicha abstracción y configurar el sentido del oído en función del reconocimiento socioespacial por el cual transita" (UA 67).

Por su parte, frente a la categoría I. A. Dimensión física $(n=3)$, solo tres de los entrevistados lograron manifestar en sus concepciones la relación desde lo físico, reconociendo en la cartografía una herramienta clave en la espacialización de fenómenos acústicos: "[...] a través de mapas de ruido o cartografías sonoras, que tienen como objetivo evaluar los niveles de ruido en un espacio específico, sobre todo en las ciudades donde se presenta más latente el fenómeno de la contaminación sonora" (UA 100).

Ahora bien, a diferencia de lo encontrado en el caso de los estudiantes, entre los maestros hubo cuatro unidades de análisis relacionadas con la dimensión holística, donde se pudieron identificar 
elementos que dieron cuenta de la interacción de las acciones humanas con lo sonoro desde la totalidad o lo sistémico:

El paisaje sonoro sería analizar una delimitación geográfica con una perspectiva bastante específica (centrarse en el estudio de determinado objeto a partir de la acepción geográfica de paisaje desde la perspectiva sonora), mientras que en lo sonoro del espacio geográfico se tendría una unidad de análisis más amplia (espacio geográfico) con un enfoque en lo sonoro (que no lo haría excluyente a otros factores que puedan intervenir). (UA 63)

Frente a la distinción paisaje sonoro de lo sonoro del espacio geográfico, un maestro manifiesta que:

El paisaje sonoro está acotando el paisaje a esa dimensión sonora que pueda contener o entrañar determinado paisaje, mientras lo sonoro del espacio geográfico es uno de los múltiples componentes de ese complejo que pueda significar el espacio geográfico tan polisémico y prolífico. (UA 87)

Una categoría adicional para el caso de los maestros, estuvo relacionada con la enseñabilidad. Esto lleva a reflexionar sobre diversas cuestiones: ¿cuáles con los contenidos que se deben enseñar?, ¿cuáles son los métodos y los recursos de apoyo didácticos más adecuados para enseñar?, ¿de qué manera evaluar lo aprendido en la enseñanza? Dicho esto, la categorización desde la didáctica dio paso a tres ejes que se convierten en subcategorías: finalidad, el ¿para qué?; contenido, el ¿qué?; y las estrategias, el ¿cómo?

Con respecto a la finalidad, hay que hacer referencia a aquellas metas definidas como enunciados que orientan los procedimientos de aprendizaje que el estudiante debe realizar en el proceso de enseñanza, las cuales son redactadas como objetivos o competencias en el marco de las unidades, estrategias o secuencias didácticas. En cuanto a este aspecto, los docentes manifestaron diversas posturas acerca del para qué llevarían al aula el estudio de lo sonoro 
del espacio geográfico: "Alcanzar una lectura diferente del espacio por medio del sonido más allá de lo que observamos, pues el escuchar nos da una comprensión más global del objeto a estudiar" (UA 73).

Con relación a las estrategias, se debe mencionar que actúan como los procedimientos (métodos, técnicas, actividades) mediante los cuales el docente organiza las acciones de manera consciente para construir y lograr metas previstas en el proceso de enseñanza. Los maestros sugirieren una serie de actividades que harían para llevar lo sonoro del espacio geográfico al aula, resaltando ejercicios enfocados en la escucha e interpretación de sonidos de diferentes paisajes, el uso de cartografías interactivas para promover formas de reproducir sonidos al tocar unas coordenadas específicas, la manipulación de la cámara y la grabadora de dispositivos móviles, donde los estudiantes a través de herramientas como GPS, Google Maps - Google Earth pudiesen registrar los sonidos y captar imágenes alusivas a estos.

Respecto al contenido, es necesario señalar que debe ser un elemento que se contenga dentro de la estructura de la finalidad y que brinde orientaciones al momento de construir las estrategias; ya que es fundamental que toda la acción de la estrategia, unidad o secuencia, esté orientada a la comprensión de los contenidos de corte declarativo, procedimental y actitudinal. De acuerdo con esto, en lo enunciado por los docentes, se destaca el tipo de contenido declarativo, a través del cual aparece la mención a distintas acepciones del espacio geográfico, resaltando las de región y paisaje.

Valdría la pena hacer referencia a la evaluación como un componente que, a través de los tipos de estrategias en las que se ponga en juego lo sonoro del espacio, posibilite justificar:

[...] qué, cómo y para qué aprenden los estudiantes y de qué manera las transformaciones suscitadas al interior del proceso en el vector tiempo y espacio, determinarán la movilidad del pensamiento, las capacidades, habilidades, actitudes y destrezas que se pondrán en juego 
en el sistema social, no solo como adaptación sino desde la acción propositiva. (Fernández et ál., 2018, p. 175)

En síntesis, desde las concepciones declaradas por los maestros, se puede inferir que asumen la relación entre lo sonoro y el espacio geográfico de manera parcial, dando predominio a las dimensiones social y subjetiva. Esto no quiere decir que deseche el aspecto didáctico que puede tener esta relación, pues valoran, de manera significativa, los aportes que se podrían generar a partir de estrategias que dinamicen procesos de enseñanza y aprendizaje desde el aula.

\section{Conclusiones}

Frente a la pregunta de investigación, se concluye que, en efecto, se pueden establecer relaciones entre lo sonoro y el espacio geográfico, más allá del concepto de paisaje sonoro, y, en esa medida, la vinculación de esta relación con la enseñanza de las ciencias sociales mediante implicaciones didácticas, desde la posibilidad del desarrollo de habilidades en la formación del pensamiento social y apuesta por la integración de las ciencias sociales.

Las concepciones declaradas por maestros y estudiantes permiten ahondar en la comprensión del sonido; pese a que es un elemento presente en la cotidianidad, comúnmente no se toma en cuenta como un aspecto susceptible de generar aportes en la manera en que habitamos y producimos el espacio. Esta situación en varios momentos remitió a la configuración cultural, dentro de la cual el sentido del oído ha sido relegado en la construcción de conocimiento.

El discurso del estudiantado otorga mayor prelación a la dimensión física, aunque, si bien se logra reconocer en lo sonoro un elemento susceptible de brindar información en torno a lo social, se evidencia cierta dificultad al momento de concebir lo sonoro más allá de la categoría de ruido. Durante el proceso investigativo, esta 
situación sugiere el contraste con otra fuente que posibilite ampliar la reflexión, momento en el que aparecen en escena los maestros, quienes desde su discurso aportan otras aristas a la discusión, lo cual se ve reflejado en la fuerza que toman categorías como lo social, lo subjetivo y lo holístico. Del mismo modo, las declaraciones de los maestros dejan entrever diversos elementos vinculados a su conocimiento profesional, lo cual logra un aporte importante para el anclaje de los saberes desde lo didáctico.

Por su parte, concebir el espacio de la ciudad desde lo sonoro como ámbito de conocimiento escolar, establece una oportunidad para abordar desde la innovación una realidad compleja, como es el fenómeno urbano. Es un marco que puede facilitar, de una manera más coherente y flexible, la integración de diferentes aportes, no solo desde lo social, sino también desde las demás áreas del conocimiento, asumiéndose, así, una capacidad de complementariedad que posibilitaría la articulación del currículo escolar, lo cual permitiría complejizar y enriquecer las posturas frente a la realidad social.

Hoy las instituciones educativas están llamadas a hacer de la ciudad y lo urbano un elemento curricular y didáctico central, como iniciativa que provea elementos para una formación ciudadana crítica frente a movimientos globalizantes, que cada vez van tomando mayor fuerza en su intención de homogenización, enmarcados bajo lógicas de formación desde la globalidad, donde lo local queda excluido. De ahí que volver a lo micro desde la comprensión del espacio como producción social resulta importante, pues la ciudad, como libro abierto de información de diversa índole, ofrece una lectura susceptible de problematizarse desde la complejidad, donde la sensibilidad y lo sensitivo toman protagonismo en una amalgama entre los objetos, la técnica y los sistemas, que, a la vez, conjuga lo social, lo físico y lo subjetivo en un holismo que permitirá poner en escena las competencias del área. 


\section{Referencias}

Alonso-Cambrón, M. (2005). Sonidoy sociabilidad: consistencia bioacústica en espacios públicos. Quaderns-e de l'Institut Català d'Antropologia, (5). https://www.researchgate.net/publication/39237943_Sonido_y_soci abilidad_consistencia_bioacustica_en_espacios_publicos

Bardin, L. (1996). El análisis de contenido (2.a edición). Akal Ediciones.

Benejam, P. (2004). Las finalidades de la educación social. En Benejam, P. y Pagés, J. (coords.) Enseñar y aprender ciencias sociales, geografía e historia en la educación secundaria (pp. 33-52). Horsori.

Boned, J. (2011). La ciudad y lo efímero. La ciudad escuchada. Ángulo Recto. Revista de estudios sobre la ciudad como espacio plural, 3(2), 5-15.

Cárdenas, R. y Martínez, D. (2015). El paisaje sonoro, una aproximación teórica desde la semiótica. Revista Investigación, Desarrollo e Innovación, 5(2), 129-140.

Cosgrove, D. (2002). Observando la naturaleza: El paisaje y el sentido europeo de la vista. Boletín de la A.G.E, (34), 63-89.

Cuervo, R. (2015). La ecología del paisaje sonoro de la ciudad: un aporte a la sostenibilidad urbana. Dearq, (16), 90-103.

Domínguez, M., Medina, A. y Sánchez, C. (2011). La Innovación en el aula: referente para el diseño y desarrollo curricular. Perspectiva Educacional, Formación de Profesores, 50(1), 61-86.

Domínguez, A. (2015). Ruido: intrusión sonora e intimidad acústica. Inmediaciones de la Comunicación, 10(10), 118-130.

Duffy, M. (2000). Lines of Drift: festival participation and performing a sense of place. Popular Music, 19(1), 51-64. 
Espinal, C. y Pulgarín, M. (2008). Dimensión Cognitiva de la geografía y su carácter integrador en la enseñanza de las Ciencias sociales. Revista Pedagógica Aulas, (5), 37-45.

Feld, S. (1996). Waterfalls of Song: An Acoustemology of Place Resounding in Bosavi, Papua New Guinea. En: Feld, S. y Basso, K. (eds.), Senses of Place (pp. 91-135). School of American Research Press.

Fernández, E., Gordo, C., López, J. y Rodríguez, A. (2019). Hacia los senderos de una evaluación transformadora: expresión del conocimiento contextual, la integración de la dimensión temporal y las posibilidades de mejores aprendizajes. Revista Interamericana de Investigación, Educación y Pedagogía (RIIEP), 2(12), 157-180.

Fortuna, C. (2009). La ciudad de los sonidos. Una heurística de la sensibilidad en los paisajes urbanos contemporáneos. Cuadernos de Antropología Social, 30, 39-58.

Garzón, J. (2017). Enseñanza de las ciencias sociales en la escuela. Pensamiento social y literatura: puntos de encuentro. Revista Noria Investigación Educativa, 1(1), 49-56.

Martínez, M. (1998). La investigación cualitativa etnográfica en educación: manual teórico práctico (3.a ed.). Trillas.

Mejía, A. y Mejía, L. (2015). Relaciones entre pensamiento histórico y pensamiento crítico en la enseñanza de las ciencias sociales en estudiantes de educación básica secundaria. Revista Interamericana de Investigación, Educación y Pedagogía (RIIEP), 2(8), 413-436.

Mejía, M. (2012). El patrimonio cultural: su gestión y significado. IBEROAMERICANO, VIII Campus de cooperación cultural. Euroamericano, VIII Campus de Cooperación Cultural. https://campuseuroamericano. org/pdf/es/ES_PATRIMONIO_CULTURAL_GESTION_SIGNIFICADO_ Mario_Mejia.pdf

Noriega, J. (2017). Análisis del campo sonoro y la molestia de la contaminación acústica en ciudades mediante el uso de redes de sensores [tesis doctoral, Universidad Católica de Murcia]. Repositorio Institucional UCAM. http://repositorio.ucam.edu/handle/10952/2883 
Pagés, J. (2007). La enseñanza de las ciencias sociales y la educación para la ciudadanía en España. Didáctica Geográfica, (9), 205-214. https:// didacticageografica.age-geografia.es/index.php/didacticageografica/ article/view/13

Pulgarín, R. (2002). El estudio del espacio geográfico, ¿posibilita la integración de las ciencias sociales que se enseñan? Revista Educación y Pedagogía, 34(14), 181 -194.

Quintero, C. y Recuero, M. (2018). El espacio urbano 'calle' a través de la mirada del paisaje sonoro. Una propuesta metodológica. Territorios, (38), 191-214.

Sánchez, A. (2015). Concepciones de los docentes en formación y sus implicaciones en el desempeño en las prácticas pedagógicas. Revista Interamericana de Investigación, Educación y Pedagogía (RIIEP), 2(8), 263-277.

Santisteban, A. (2011). Las finalidades de la enseñanza de las Ciencias sociales. En Pagés. J. y Santisteban. A (coords.), Didáctica del conocimiento del medio social y cultural en la educación primaria (pp. 63-70). Editorial Síntesis.

Schafer, M. (1969). El nuevo paisaje sonoro. Ricordi Americana S.A.

Sui, D. (2000). Visuality, Aurality, and Shifting Metaphors of Geographical Thought in the Late Twentieth Century. Annals of the Association of American Geographers, 90(2), 322-343.

Stake, R. (2005). Investigación con estudio de casos (3. a ed.). Ediciones Morata.

Zigor, M. (2017). Una aproximación perceptiva al entorno sonoro urbano, variante metodológica en el Casco Viejo de Bilbao [tesis doctoral, Universidad del País Vasco]. https://doi.org/10/24047 